\title{
Polymerization Behavior of Poly(ether ketone) via Friedel-Crafts Acylation Studied by End-Group Analysis with ${ }^{1}$ H NMR
}

\author{
Yoshimitsu SaKaguchi, Kunio Kimura, ${ }^{*}$ Manabu OMORI, ${ }^{*}$ \\ and Yuhiko YAMASHITA* \\ Toyobo Research Center Co., Ltd., 1-1 Katata 2-Chome, Ohtsu 520-0292 Japan \\ *Faculty of Environmental Science and Technology, Okayama University, \\ 3-1-1 Tsushima-naka, Okayama 700-8530 Japan
}

(Received November 29, 2001; Accepted February 5, 2002)

\begin{abstract}
Polymerization process of poly(ether ketone) made of diphenyl ether (DPE) and isophthaloyl chloride (IPC) catalyzed by aluminum chloride in dichloroethane was characterized by ${ }^{1} \mathrm{H}$ NMR. The polymerization gave a precipitate of polymer/catalyst complex during the reaction. End-group analyses for polymer appeared as precipitate and oligomers stayed in the solution were carried out respectively after decomposition of the complex by treatment with methanol. Time dependence of the content of end groups showed that the oligomer of which molecular weight exceeded a critical value precipitated out independent of the functionality of the end groups, and further reaction proceeded in the precipitate as well as in the solution. Another approach to understand the reaction was carried out by direct ${ }^{1} \mathrm{H}$ NMR observation of the polymerization in an NMR sample tube. This experiment detected the molecular weight change of soluble oligomer in the reaction system at real time, and the results were consistent with those of isolated oligomers described above.

KEY WORDS Poly(ether ketone) / Friedel-Crafts Acylation / End-Group Analysis / ${ }^{1} \mathrm{H}$ NMR / Polymer-Catalyst Complex / Precipitate /
\end{abstract}

Poly(arylene ether ketone)s (PEKs) are well known as high performance semicrystalline polymers having excellent thermal stability, mechanical properties and solvent resistance.

Preparations of PEKs are classified into two methods. ${ }^{1-3}$ One is the aromatic nucleophilic substitution reaction between activated aromatic halides and alkali metal phenoxides in which ether linkages are formed. This method is used for the production of not only poly(ether ether ketone) (PEEK), most popular representative of PEKs, but also commercial polysulfones and poly(ether sulfone)s. The other method is the aromatic electrophilic substitution reaction between electron-rich aromatic hydrocarbons and aromatic acid chlorides in which ketone linkages are formed. This polymerization is carried out either in an organic solvent with aluminum chloride as a catalyst, ${ }^{4,5}$ FriedelCrafts acylation, or in hydrogen fluoride/boron trifluoride as a catalyst and solvent. 6,7 The polymerization also includes dehydrative acylation reaction in polyphosphoric acid, ${ }^{8,9}$ in trifluoromethanesulfonic acid $^{10}$ and in a methanesulfonic acid/phosphorus pentoxide medium. ${ }^{11}$

Preparation of poly(ether ketone)s with FriedelCrafts acylation catalyzed by aluminum chloride in dichloroethane as a solvent can be carried out under moderate conditions, and is considered as a convenient method to obtain poly(ether ketone)s on a laboratory scale. Furthermore, various combinations of monomers afford a number of structures of polymers.

The polymerization by Friedel-Crafts acylation gives a precipitate of the complex of polymer and catalyst in the course of polymerization. ${ }^{12}$ It has been revealed that the molecular weight of PEK increases in the precipitate with reaction time. ${ }^{13}$ Since it is generally thought that precipitation during polymerization terminates the chain growth, the increase in molecular weight after precipitation is very interesting phenomenon. In general, solution polycondensation gives higher molecular weight when the reaction is carried out at a higher monomer concentration. On the contrary, this polymerization has a tendency to give higher molecular weight when the reaction is carried out at a relatively lower monomer concentration. Moreover, influence of monomer stoichiometric ratio on the molecular weight of resultant polymer is another characteristic phenomenon; it was reported that the polymerization with excess of DPE gave relatively higher molecular weight polymer than that with excess of IPC did. ${ }^{14}$

As described above, the polymerization of PEK by Friedel-Crafts acylation has several characteristics different from general polycondensation reactions. Therefore, understanding of the polymerization mechanism of PEK with phase separation, especially the reaction mechanism in the precipitate, is very important and may lead us to control the polymerization process and the morphology of resultant polymers. Change in the inherent viscosity of polymer in the precipitate and 
oligomer in the solution with reaction time was chased in the past, ${ }^{13,14}$ however, further investigation has not yet been investigated. In this study, the polymerization of PEK by Friedel-Crafts acylation was characterized mainly by using NMR method.

\section{EXPERIMENTAL}

\section{Materials}

Isophthaloyl chloride (IPC, Mitsubishi Gas Chemical Co., Inc) and Aluminum chloride $\left(\mathrm{AlCl}_{3}\right.$, Nacalai Tesque, EP) were ground to powders in nitrogen atmosphere. 1,2-Dichloroethane (DCE, Nacalai Tesque, GR) was dried over molecular sieves. Diphenyl ether (DPE, Tokyo Kasei) was distilled under vacuum before use.

\section{Polymerization}

A standard procedure for the preparation of PEK is as follows. In a $300 \mathrm{~mL}$ of four-necked flask equipped with a nitrogen inlet and a stirring rod, $12.22 \mathrm{~g}$ of DPE $(0.07182 \mathrm{~mol})$ and $14.58 \mathrm{~g}$ of IPC $(0.07182 \mathrm{~mol})$ were dissolved in $200 \mathrm{~mL}$ of DCE. After the solution was cooled below $5^{\circ} \mathrm{C}$ in an ice bath, $24.96 \mathrm{~g}$ of $\mathrm{AlCl}_{3}$ $(0.1872 \mathrm{~mol})$ was added, and the reaction mixture was stirred for $2 \mathrm{~h}$. Thereafter, the reaction was continued at room temperature for $21 \mathrm{~h}$. The precipitate was separated by filtration after the reaction, and the solvent was removed by evaporation. The oligomer dissolved in the solution was collected from the filtrate by evaporation of the solvent. The powders of precipitate and oligomer thus obtained were soaked in methanol to decompose the catalyst-product complex and then washed with methanol by using soxhlet. The samples thus obtained were dried at $150^{\circ} \mathrm{C}$ under vacuum for $17 \mathrm{~h}$.

The samples to be characterized were obtained with different reaction times.

\section{Characterization}

Inherent viscosities were measured with an Ostwald type viscometer at a concentration of $0.1 \mathrm{~g} \mathrm{dL}^{-1}$ in $97 \%$ sulfuric acid at $30^{\circ} \mathrm{C} .{ }^{1} \mathrm{H}$ NMR spectra for the isolated products were obtained on a Varian Unity-500 spectrometer in a mixture of $\mathrm{CDCl}_{3}$ and trifluoroacetic acid at room temperature. Direct observation of PEK polymerization in a NMR tube was carried out with a sealed capillary containing deuterated benzene.

\section{RESULTS AND DISCUSSION}

\section{End-Group Analysis by ${ }^{1} H$ NMR}

In this study, polymerization of diphenyl ether (DPE) and isophthaloyl chloride (IPC) in dichloroethane cat-
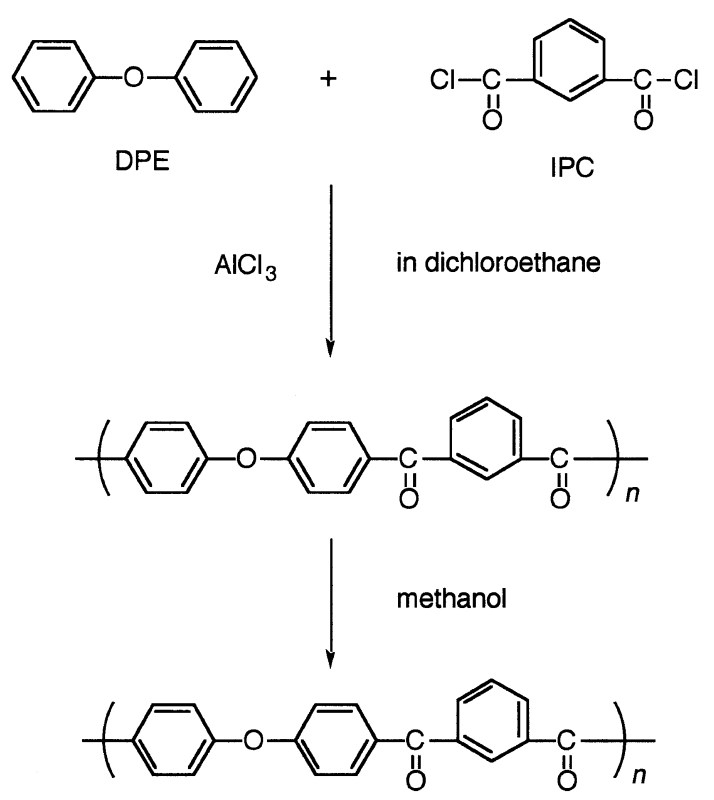

Scheme 1.

alyzed by aluminum chloride was chosen to investigate the polymerization behavior of PEK by Friedel-Crafts acylation (Scheme 1). Firstly, PEKs were prepared with varying the feed ratio of IPC and DPE to detect the end groups of polymer chain by ${ }^{1} \mathrm{H}$ NMR. Figure 1 shows ${ }^{1} \mathrm{H}$ NMR spectra of resulting PEKs with different monomer feed ratios. The stoichiometric feed gave a polymer with increased molecular weight, so that the signals of chain ends were hardly detected in the spectrum. Signals of IPC terminal were clearly observed for the polymer obtained from the polymerization with excess of IPC, and those of DPE terminal were with excess of DPE. These results show that the polymerization of PEK can be followed by the end-group analysis with ${ }^{1} \mathrm{H}$ NMR.

${ }^{1} \mathrm{H}$ NMR spectrum of PEK oligomer obtained at an early stage of the polymerization is shown in Figure 2. Signals were assigned with a help of the COSY spectrum as shown in Figure 3. Signals of IPC at the chain end and next to the DPE end group are distinguished from those of internal IPC units in the polymer chain. Signals of the two DPE units from the chain end are at different positions from other DPE units. A signal at $4 \mathrm{ppm}$ is assigned to the protons of methyl ester formed by treatment of the products with methanol to decompose the polymer/aluminum chloride complex. From signal intensity, it is considered that most of the unreacted carbonyl chlorides changed to methyl esters.

Time Dependence of End-Group Ratio in the Process of Polymerization

Polymerization of PEK by Friedel-Crafts acylation in which the precipitate of polymer/aluminum chloride complex appeared was investigated at a polymer con- 
Table I. Time dependence of the yield and inherent viscosity of PEK

\begin{tabular}{|c|c|c|c|c|c|}
\hline Reaction time & \multicolumn{2}{|c|}{ Polymer precipitate } & \multicolumn{2}{|c|}{ Soluble oligomer } & Total yield \\
\hline $\mathrm{h}$ & $\frac{\text { Yield }}{\%}$ & $\frac{\eta_{\text {inh }}}{\mathrm{dL} \mathrm{g}^{-1}}$ & $\frac{\text { Yield }}{\%}$ & $\frac{\eta_{\text {inh }}}{\mathrm{dL} \mathrm{g}^{-1}}$ & $\%$ \\
\hline 0.67 & 37.3 & 0.20 & 32.5 & 0.11 & 69.8 \\
\hline 0.95 & 68.4 & 0.21 & 25.1 & 0.10 & 93.5 \\
\hline 1.08 & 77.5 & 0.32 & 18.8 & 0.11 & 96.3 \\
\hline 1.50 & 86.3 & 0.42 & 6.6 & 0.09 & 92.9 \\
\hline 2.25 & 94.6 & 0.71 & 3.1 & 0.12 & 97.7 \\
\hline 12 & 95.8 & 0.74 & 0.7 & 0.15 & 96.2 \\
\hline 21 & 99.9 & 0.67 & 0.5 & 0.05 & 100.4 \\
\hline
\end{tabular}

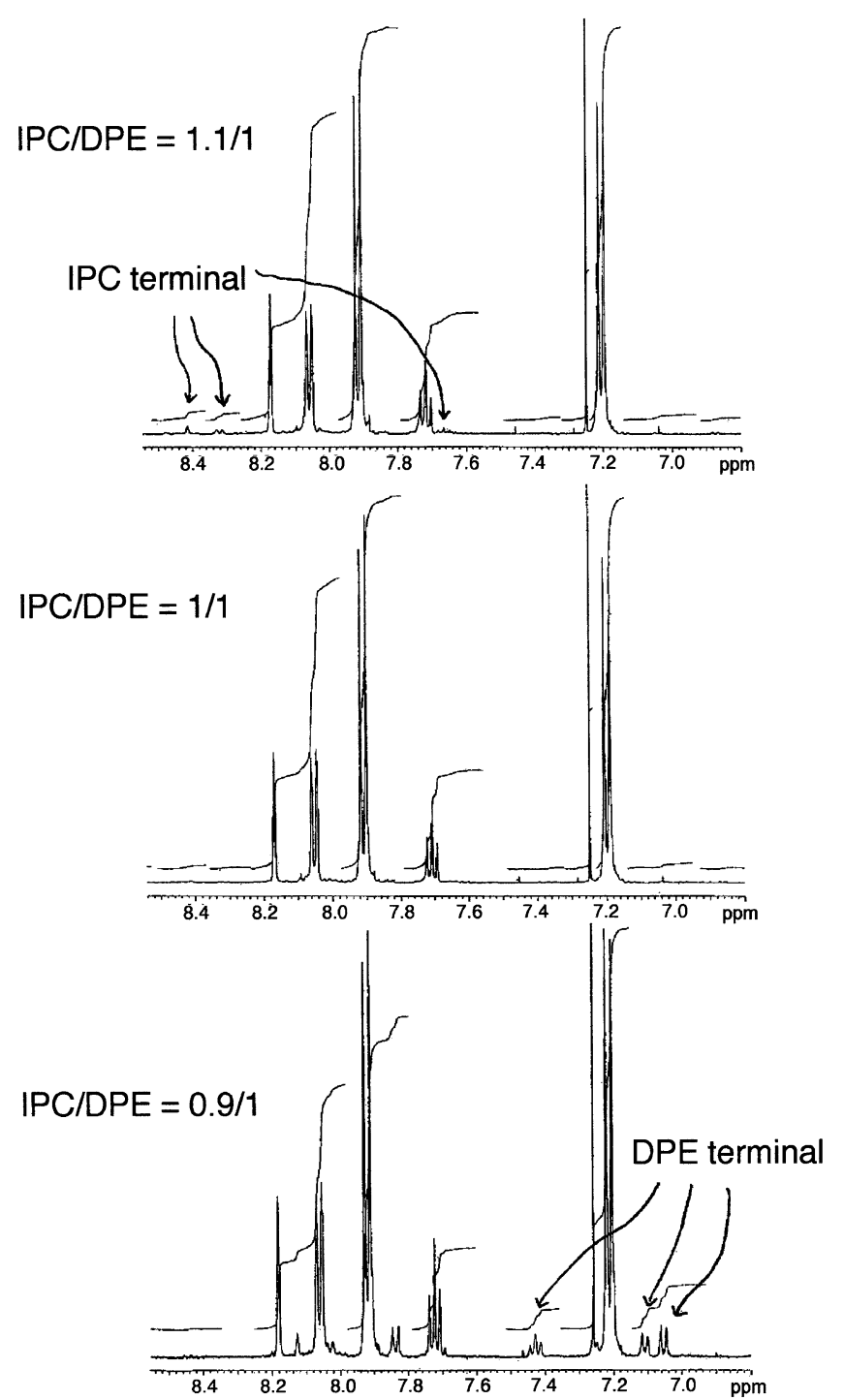

Figure 1. ${ }^{1} \mathrm{H}$ NMR spectra of resulting PEKs with different monomer feed ratios.

centration of $8 \mathrm{wt} \%$ and 2.6 molar equivalents of aluminum chloride relative to IPC. The complex began to precipitate after $30 \mathrm{~min}$ of polymerization, and then the size and amount of precipitate increased with time. The polymerizations were stopped at different reaction times to obtain the samples to be analyzed. The polymer precipitated and the oligomer recovered from the solution were treated with methanol to decompose the complex with aluminum chloride, respectively.

Table I shows change in the yield and inherent viscosity of the precipitate and the oligomer with reaction time. As reported earlier, ${ }^{13}$ the yield of precipitate increased with time up to $2 \mathrm{~h}$ and then it became constant. The inherent viscosity of the precipitate changed in a similar manner to the yield. On the other hand, the oligomer dissolved in solution decreased with reaction time, while the inherent viscosity was constant around $0.1 \mathrm{dL} \mathrm{g}^{-1}$. These results indicate that the oligomers of which molecular weight exceeds a critical value precipitate out, and further reaction proceeds in the precipitate.

${ }^{1} \mathrm{H}$ NMR analysis was carried out for the samples above as shown in Table II. Contents of DPE- and IPC-unit in each sample were almost equivalent. Concerning the end groups, however, there was a tendency that less IPC end-group than DPE was detected, and this tendency was more remarkable at the early stage of polymerization and for the oligomer. This is because lower molecular weight oligomers having IPC end-group, which had changed to methyl ester, were easily washed out by methanol treatment. When the end-group analysis was carried out including components in the washings, it was confirmed that the both end groups were almost equivalent. Therefore, the precipitation is caused by the increase in molecular weight of the oligomer irrespective of the kind of end groups, DPE terminal or IPC terminal.

The number average degree of polymerization $\left(\mathrm{DP}_{\mathrm{n}}\right)$ of PEK was estimated with the content of end-groups by the following equation:

$$
\begin{aligned}
\mathrm{DP}_{\mathrm{n}}= & 200 /([\mathrm{DPE} \text { end-group }(\%)] \\
& +[\text { IPC end-group }(\%)])
\end{aligned}
$$

Time dependence of the $\mathrm{DP}_{\mathrm{n}}$ with reaction thus estimated is shown in Figure 4. The result is in good agreement with that of inherent viscosity. Changes in the $\mathrm{DP}_{\mathrm{n}}$ and the yield with reaction time for the precipitate and the oligomer are compared in Figure 5. These results indicate that the oligomers of which molecular weight exceeds a critical value precipitate out, and 

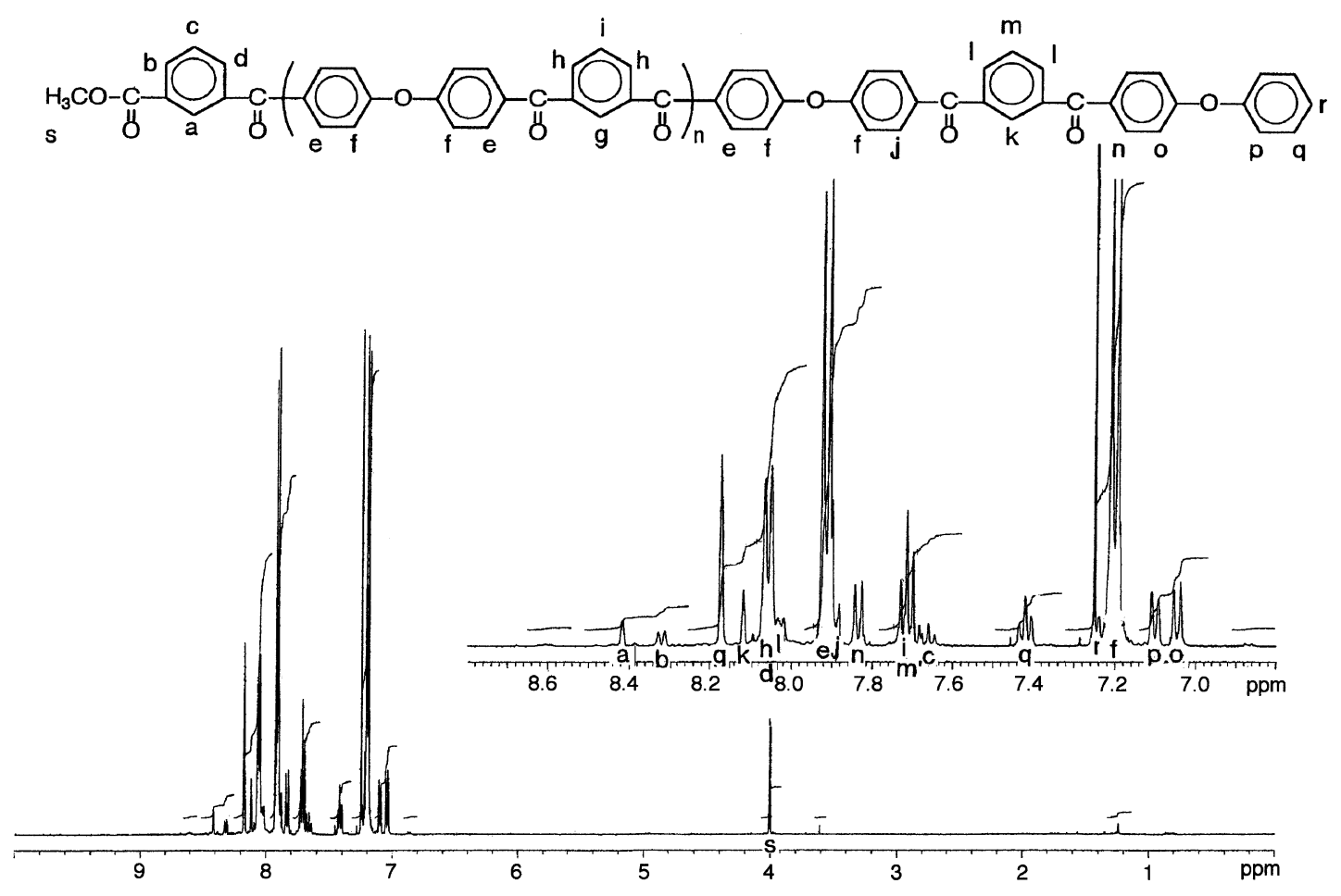

Figure 2. ${ }^{1} \mathrm{H}$ NMR spectrum of a low molecular weight PEK.

Table II. Time dependence of the end-group analysis of PEK

\begin{tabular}{|c|c|c|c|c|c|c|}
\hline \multirow{2}{*}{$\frac{\text { Reaction time }}{\mathrm{h}}$} & \multicolumn{3}{|c|}{ Polymer precipitate } & \multicolumn{3}{|c|}{ Soluble oligomer } \\
\hline & $\begin{array}{l}\text { DPE-unit } / \%^{\mathrm{a}} \\
\text { (end ratio } / \%^{\mathrm{b}} \text { ) }\end{array}$ & $\begin{array}{c}\text { IPC-unit }^{\mathrm{a}} \\
\left(\text { end ratio } / \%{ }^{\mathrm{b}}\right)\end{array}$ & $\mathrm{DP}^{\mathrm{c}}$ & $\begin{array}{l}\text { DPE-unit } / \%^{\mathrm{a}} \\
\left(\text { end ratio/\% } \%{ }^{\mathrm{b}} \text { ) }\right.\end{array}$ & $\begin{array}{c}\text { IPC-unit }^{\mathrm{a}} \\
\left(\text { end ratio/\% } \%{ }^{\mathrm{b}}\right)\end{array}$ & $\mathrm{DP}^{\mathrm{c}}$ \\
\hline 0.67 & $37.3(12)$ & $37.3(9)$ & 9.5 & $32.5(25)$ & $31.5(12)$ & 5.4 \\
\hline 0.95 & $68.4(12)$ & $67.0(7)$ & 10.5 & $25.1(26)$ & $23.6(10)$ & 5.6 \\
\hline 1.08 & $77.5(6)$ & $76.7(7)$ & 15.4 & $18.8(24)$ & $18.8(16)$ & 5.0 \\
\hline 1.50 & $86.3(3)$ & $84.5(5)$ & 25.0 & $6.6(26)$ & $6.1(20)$ & 4.3 \\
\hline 2.25 & $94.6(\sim 0)$ & $94.6(4)$ & 50.0 & $3.1(11)$ & $2.9(22)$ & 6.1 \\
\hline 12 & $95.8(\sim 0)$ & $95.8(3)$ & 66.7 & - & - & - \\
\hline 21 & $99.9(\sim 0)$ & $99.9(4)$ & 50.0 & - & - & - \\
\hline
\end{tabular}

\footnotetext{
${ }^{\mathrm{a}}$ Yield of monomer-unit relative to the feed (\%). ${ }^{\mathrm{b}}$ End-group ratio relative to total monomer unit (\%). ${ }^{\mathrm{c}}$ Degree of polymerization calculated based on end-group analysis.
}

further reaction proceeds in the precipitate. As the $\mathrm{DP}_{\mathrm{n}}$ of oligomers was around 5, it is considered that the oligomer begins to precipitate when the molecular weight becomes at least higher than $c a .1500$ as the isolated polymer, or $c a$. 2800 as the polymer/catalyst complex.

From these results, the following reaction mechanism is suggested. When the $\mathrm{DP}_{\mathrm{n}}$ of oligomers exceeds the critical value in the course of polymerization, they begin to precipitate due to immiscibility with the solvent. In the precipitate, further polymerization continues between oligomers because the precipitate swollen by the solvent has mobility to react each other. Continuous supply of the oligomers into the precipitate helps the precipitate to increase the $\mathrm{DP}_{\mathrm{n}}$. After the $\mathrm{DP}_{\mathrm{n}}$ increases to a certain level and the oligomer supply is exhausted, the polymerization in the precipitate ceases due to the lack of mobility and decrease in the content of reactive end groups.

\section{Direct Observation of PEK Polymerization in NMR Tube}

Characterization of polymers and oligomers after decomposition of the complex may not be a real observation of the polymerization itself. From this point of view, polymerization between DPE and IPC in dichloroethane at a concentration of $8 \mathrm{wt} \%$ was carried out in an NMR sample tube, and ${ }^{1} \mathrm{H}$ NMR spectrum was recorded directly to detect signals of the oligomers in solution. In this measurement, there is no fear of effusion of very low molecular weight oligomer by methanol treatment to isolate the soluble oligomers, although a disadvantage for the quantitative analysis arises from the broadness of signals compared to the 


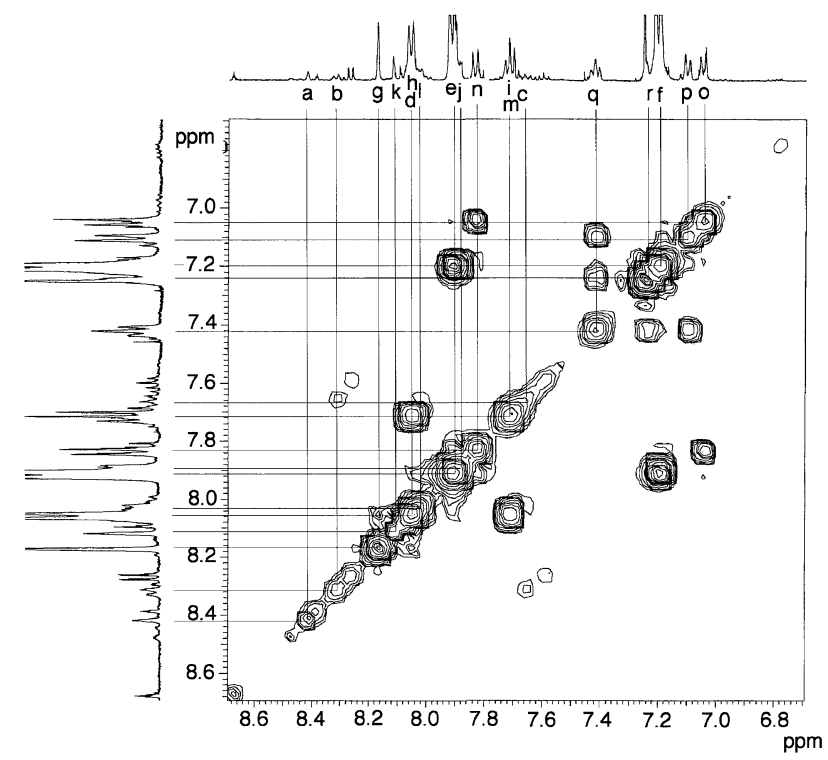

Figure 3. ${ }^{1} \mathrm{H}$ COSY spectrum of a low molecular weight PEK.

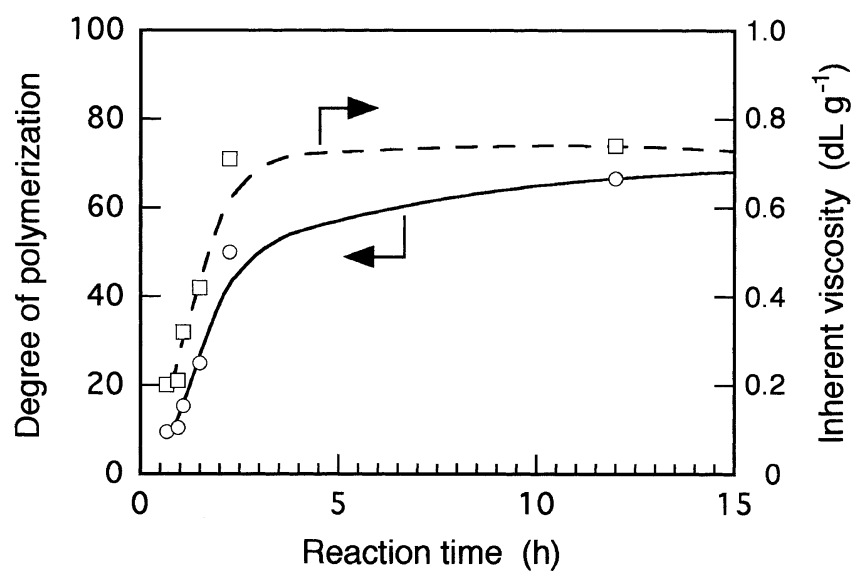

Figure 4. Change in the degree of polymerization estimated by NMR analysis and the inherent viscosity for the polymers precipitated.

\section{isolated oligomers.}

Figure 6 shows the spectral change in ${ }^{1} \mathrm{H}$ NMR of polymerization system with reaction time. Although the spectrum pattern was somewhat different from that of the isolated oligomers, DPE terminal (ortho protons of ether) was estimated by using signals at $7 \mathrm{ppm}$, and IPC terminal (ortho protons of carbonyl) at 8.5$9.0 \mathrm{ppm}$. Intensities of the signals decreased as the polymerization proceeded. This means that the concentration of oligomers became lower due to the precipitation.

As seen in Figure 6, very broad signals due to a part of swelled precipitate were detected after $4 \mathrm{~h}$. The precipitate appeared irregularly on the wall of sample tube and did not give quantitative information. Therefore, the degree of polymerization for soluble oligomers until $6 \mathrm{~h}$ was estimated as shown in Figure 7. The degree of polymerization increased to around four with reaction
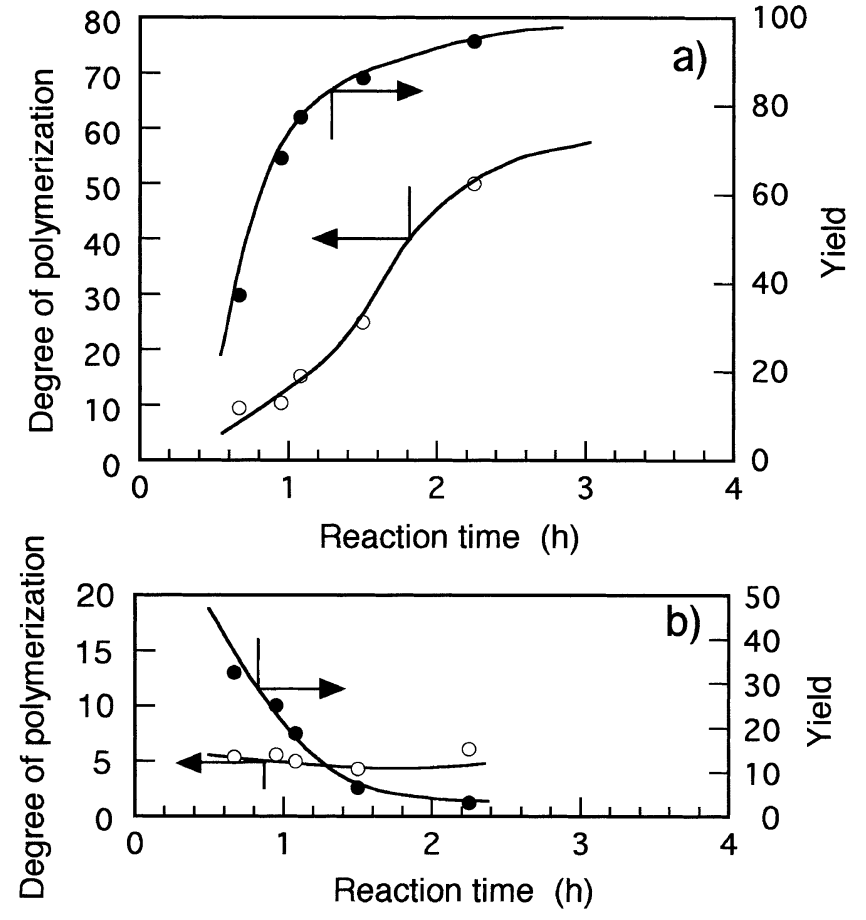

Figure 5. Change in the degree of polymerization and the yield for a) the precipitate and b) the oligomer recoverd from solution.

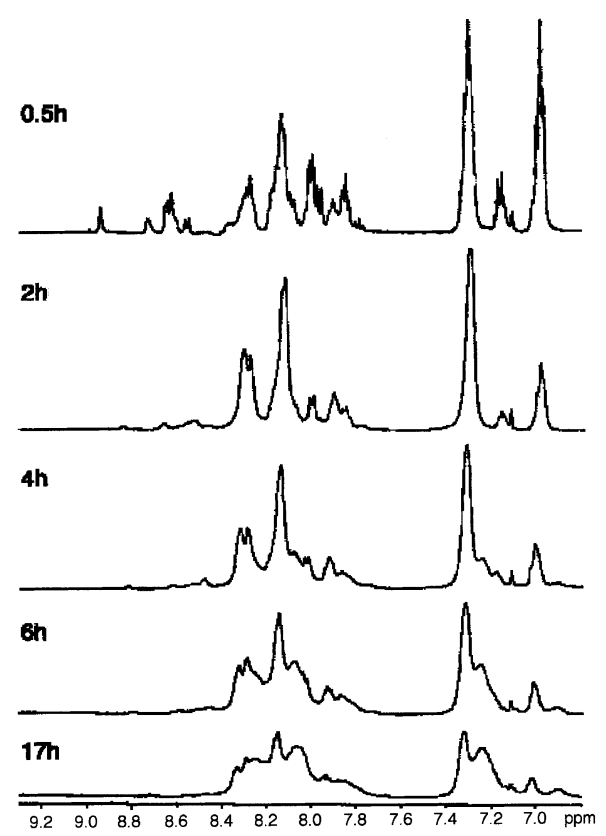

Figure 6. ${ }^{1} \mathrm{H}$ NMR change for the polymerization in NMR tube.

time, then leveled off. These results were generally in agreement with the results obtained from the isolated oligomers from the reaction supernatant, because the constant $\mathrm{DP}_{\mathrm{n}}$ obtained for the isolated oligomers were the results of extraction of very low molecular weight oligomers and monomers by methanol.

Process of precipitation polymerization of PEK via Friedel-Crafts acylation was successfully characterized by using NMR in this study. The results gave useful 


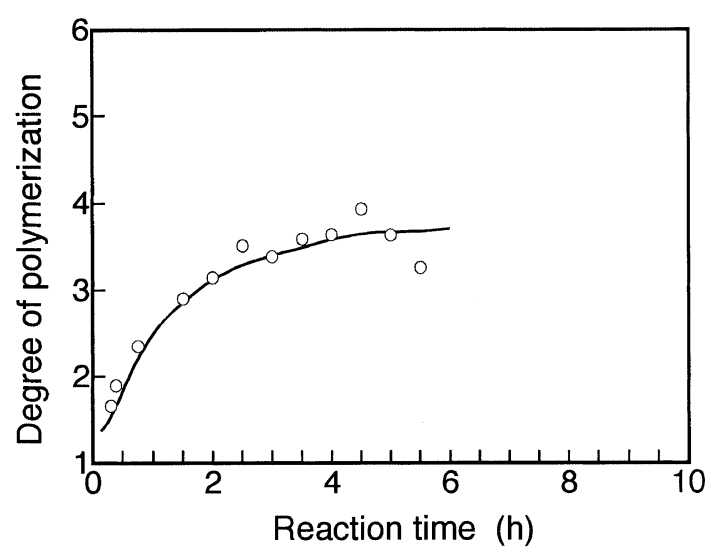

Figure 7. Time dependence of the degree of polymerization for the soluble oligomer in the reaction mixture directly observed by ${ }^{1} \mathrm{H}$ NMR.

information to clarify the polymerization mechanism, especially in the early stages that the precipitation occurred. Further study on the mechanism focused on the reaction within the precipitate is now in progress.

\section{CONCLUSION}

End group analysis by ${ }^{1} \mathrm{H}$ NMR for the precipitated polymers and soluble oligomers at various reaction times clarified the change in molecular weight and functionality of the chain ends. Direct ${ }^{1} \mathrm{H}$ NMR observation of the polymerization reaction performed in a NMR tube also detected the change in the molecular weight of soluble oligomer with time. The results showed that the oligomers of which molecular weight exceeds a critical value, the $\mathrm{DP}_{\mathrm{n}}$ around 5 , precipitate out, and further reaction proceeds in the precipitate with continuous supply of the oligomers into the precipitate. The precipitation was caused by the increase in molecular weight of the oligomer irrespective of the kind of end groups, DPE terminal or IPC terminal.

\section{REFERENCES}

1. J. B. Rose, "High Performance Polymers: Their Origin and Development”, R. B. Seymour and G. S. Kirshenbaum, Ed., Elsevier Science Inc., New York, N.Y., 1986, p 187.

2. M. J. Mullins and E. P. Woo, J. Macromol. Sci., Rev. Macromol. Chem. Phys., C27, 313 (1987).

3. V. L. Rao, J. Macromol. Sci., Rev. Macromol. Chem. Phys., C35, 661 (1995).

4. W. H. Bonner, U. S. Patent 3442857 (1969).

5. I. Goodman, J. E. McIntyre, and W. Russell, British Patent 971227 (1964).

6. B. M. Marks, U. S. Patent 3442857 (1969).

7. V. Jansons and K. Dahl, Makromol. Chem., Macromol. Symp., 51, 87 (1991).

8. Y. Iwakura, K. Uno, and T. Takiguchi, J. Polym. Sci., A-1, 6, 3345 (1968).

9. Y. Iwakura, K. Uno, and T. Takiguchi, J. Polym. Sci., Polym. Lett. Ed., 15, 283 (1977).

10. W. Risse, D. Y. Sogah, and F. P. Boettcher, Makromol. Chem., Macromol. Symp., 44, 185 (1991).

11. M. Ueda and M. Sato, Macromolecules, 20, 2675 (1987).

12. M. G. Zolotukhin, N. G. Gileva, S. N. Salazkin, Y. A. Sangalov, Y. V. Genin, and V. S. Sultanova, Polym. Sci.. USSR, 31, 2748 (1989).

13. Y. Sakaguchi, M. Tokai, and Y. Kato, Polymer, 34, 1513 (1993).

14. M. G. Zolotukhin, D. R. Rueda, F. J. Balta Calleja, M. E. Cagiano, M. Bruix, and E. A. Sedova, Polymer, 38, 1471 (1997). 